Proceedings of the 1997 IEEE

International Conference on Robotics and Automation

Albuquerque, New Mexico - April 1997

\title{
Stable Poses of 3-Dimensional Objects
}

\author{
Richard Mason \\ Dept. of Mechanical Engineering \\ California Inst. of Technology \\ Pasadena, CA 91125
}

\author{
Elon Rimon \\ Dept. of Mechanical Engineering \\ Technion, Israel Inst. of Technology \\ Haifa, ISRAEL
}

\author{
Joel Burdick \\ Dept. of Mechanical Engineering \\ California Inst. of Technology \\ Pasadena, CA 91125
}

\begin{abstract}
This paper considers the gravitational stability of a frictionless 3-dimensional object in contact with and arbitrarily curved objects. This paper also shows how to determine the region over which the object's center of mass can move while the object maintains a given set of contacts and remains in stable equilibrium. We present symbolic solutions for up to three contacts and discuss numerical solutions for larger numbers of contacts. This analysis has application in planning the motions of quasi-statically walking robots over uneven terrain and the manipulation of heavy objects.
\end{abstract}

\section{Introduction}

We consider a rigid object, $\mathcal{B}$, in frictionless contact with one or more rigid and immovable bodies, $\mathcal{A}_{1}, \ldots, \mathcal{A}_{k}$, in the presence of a conservative force, such as gravity. When will a posture of $\mathcal{B}$ be stable? In Ref. [1], the authors presented the most general conditions for stable equilibrium, taking into account the surface curvature of the contacting bodies. We also considered in detail the stability of planar objects. This paper extends that work to the case of 3-dimensional (3D) solid objects in a uniform gravitational field. In particular, we develop a procedure to find, for a given contact geometry, the stable positions of $\mathcal{B}$ 's center of mass.

Our analysis has several practical applications. If the obstacles are robot "fingers" in contact with $\mathcal{B}$, then our problem amounts to determining whether the fingers stably support $\mathcal{B}$ in the presence of gravity. Our analysis may also be useful for studying the stability of a multi-limbed robot which is moving over convoluted terrain, where the supporting obstacles are interpreted as "footholds." If the robot moves quasi-statically, then at any given moment it can be considered as a single rigid body and the question of whether it slips and falls is the same as the problem considered here. The robot may want to change its posture while maintaining all of its supporting footholds so as to establish a new foothold. We would like to know exactly how far the robot can move its center of mass without losing its stable stance. We establish means to compute the boundaries of the center of mass location which ensure stability. These results are in some sense a generalization of the well-known "tripod rule" which is used for planning quasistatic multi-legged locomotion over flat terrain. While we assume frictionless contacts, some friction will be present in these applications. Nevertheless, our frictionless analysis will be maximally conservative.

\section{Relation to Previous Work}

Robotics researchers have considered the stability of an object under gravity in the context of sensorless part manipulation [2], quasi-static manipulation [4], and object recognition [5]. However, these analyses considered a single rigid object lying on a flat plane. In our analysis, the contacts need not lie in a plane and can assume any curvature. We only require that the boundary of $\mathcal{B}$ is smooth in the vicinity of the contacts. In the context of "whole arm manipulation," Trinkle et al. [6] have also extensively studied the stability of a multiply contacted body under gravity. Our approach extends many of their results, considering non-polyhedral objects and investigating the region of stability with respect to displacements of the object's center of mass.

\section{Background/Review}

This section reviews our notation (which closely follows Ref. [1]) and basic concepts. The stability of $\mathcal{B}$ with respect to the stationary supporting bodies $\mathcal{A}_{1}, \ldots, \mathcal{A}_{k}$ can be analyzed in $\mathcal{B}$ 's configuration space (or $c$-space), denoted $\mathcal{C}$. Configurations $q \in \mathcal{C}$ are given hybrid coordinates $q=(d, \theta)$ where $d \in \mathbb{R}^{3}$ parametrizes $\mathcal{B}$ 's position, and $\theta \in \mathbb{R}^{3}$ parametrizes orientation via the exponential map. From $\mathcal{B}$ 's perspective, the supporting bodies form stationary "obstacles." The c-space obstacle (or cobstacle) corresponding to $\mathcal{A}_{i}$, denoted $\mathcal{C A}_{i}$, is the set of $\mathcal{B}$ 's configurations where it intersects $\mathcal{A}_{i}$. The boundary of $\mathcal{C} \mathcal{A}_{i}$, denoted $\mathcal{S}_{i}$, consists of configurations where $\mathcal{B}$ touches $\mathcal{A}_{i}$ such that their interiors are disjoint. If $\mathcal{B}$ is supported by $k$ bodies, $q_{0}$ lies on the intersection of $\mathcal{S}_{i}$ for $i=1, \ldots, k$. At the configuration $q_{0}, \mathcal{B}$ is in contact with $\mathcal{A}_{i}$ at a point $x_{i}=R(\theta) r_{i}+d$ where $r_{i}$ is the description of the contact point in $\mathcal{B}$ 's body coordinates. The tangent space to $\mathcal{S}_{i}$ at $q_{0}$ is denoted $T_{q_{0}} \mathcal{S}_{i}$, and the tangent space to any $q \in \mathcal{C}$ is denoted $T_{q_{0}} \mathcal{C}$.

The free configuration space (or freespace $\mathcal{F}$ ) is the complement of the interior of the c-obstacles. The free motions of $\mathcal{B}$ at a contact configuration $q_{0}$ are those curves that emanate from $q_{0}$ and locally lie in $\mathcal{F}$. These curves 
determine the local motions of $\mathcal{B}$ along which it either breaks away from or maintains surface contact with the supporting bodies. The tangent vectors to the free motions at $q_{0}$ are called the $1^{\text {st }}$ order free motions of $\mathcal{B}$ at $q_{0}$. Let $n_{i}\left(q_{0}\right)$ be the outward pointing unit normal to $\mathcal{S}_{i}$ at $q_{0}$. Let $\hat{l}_{i} \in \mathbb{R}^{3}$ be the outward pointing unit normal to $\mathcal{A}_{i}$ at $x_{i}$. Let $\rho_{i}(\theta)=R(\theta) r_{i}$. Then $n_{i}\left(q_{0}\right)=\frac{1}{c_{i}}\left(\hat{l}_{i}, \rho_{i}(\theta) \times \hat{l}_{i}\right)^{T}$ where $c_{i}=\left\|\left(\hat{l}_{i}, \rho_{i}(\theta) \times \hat{l}_{i}\right)^{T}\right\|$. The positive span of all the $n_{i}$ is the normal cone at $q_{0}$, denoted by $N\left(q_{0}\right)$.

$$
N\left(q_{0}\right)=\left\{\sum_{i=1}^{k} \lambda_{i} n_{i}\left(q_{0}\right): \lambda_{i} \geq 0 \quad \forall i\right\}
$$

We will subsequently need an expression for $\kappa_{i}(q, \dot{q})$, the curvature of the $i^{\text {th }}$ c-obstacle boundary, $\mathcal{S}_{i}$, at a point $q \in \mathcal{S}_{i}$. Let $L_{\mathcal{B}_{i}}$ and $L_{\mathcal{A}_{i}}$ respectively denote the curvatures of $\mathcal{B}$ and $\mathcal{A}_{i}$ at their contacting point, $x_{i}$.

Lemma 3.1 ([7]) Let $\mathcal{B}(q)$ contact $\mathcal{A}_{i}$ at a point $x_{i}=$ $R(\theta) r_{i}+d$. The c-space curvature of $\mathcal{S}_{i}$ at $q \in \mathcal{S}_{i}$ is

$$
\begin{gathered}
\kappa_{i}(q, \dot{q})=\dot{q}^{T} D n_{i}(q) \dot{q}=\frac{1}{c_{i}}\left(v^{T} \omega^{T}\right)\left\{\left[\begin{array}{cc}
I & -\left[\rho_{i} \times\right] \\
0 & {\left[\hat{l}_{i} \times\right]}
\end{array}\right]^{T}\right. \\
{\left[\begin{array}{cc}
L_{\mathcal{B}_{i}}\left[L_{\mathcal{A}_{i}}+L_{\mathcal{B}_{i}}\right]^{-1} L_{\mathcal{A}_{i}} & -L_{\mathcal{A}_{i}}\left[L_{\mathcal{A}_{i}}+L_{\mathcal{B}_{i}}\right]^{-1} \\
-\left[L_{\mathcal{A}_{i}}+L_{\mathcal{B}_{i}}\right]^{-1} L_{\mathcal{A}_{i}} & -\left[L_{\mathcal{A}_{i}}+L_{\mathcal{B}_{i}}\right]^{-1}
\end{array}\right]} \\
\left.\left[\begin{array}{cc}
I & -\left[\rho_{i} \times\right] \\
0 & {\left[\hat{l}_{i} \times\right]}
\end{array}\right]+\left[\begin{array}{cc}
0 & 0 \\
0 & -\left(\left[\rho_{i} \times\right]^{T}\left[\hat{l}_{i} \times\right]\right)_{s}
\end{array}\right]\right\}\left(\begin{array}{c}
v \\
\omega
\end{array}\right) \\
\text { where } \dot{q}=(v, \omega)^{T} \in T_{q} \mathcal{S}_{i} \text { and } c_{i}=\sqrt{1+\left\|\rho_{i} \times \hat{l}_{i}\right\|^{2}}
\end{gathered}
$$

For a given matrix $A, A_{s}=\frac{1}{2}\left(A+A^{T}\right)$, and $[a \times]$ denotes the skew-symmetric matrix such that $[a \times] b=a \times b$ for all $a, b \in \mathbb{R}^{3}$. It is always true that $\left(L_{\mathcal{A}_{i}}+L_{\mathcal{B}_{i}}\right) \geq 0$, otherwise the two surfaces would interpenetrate at the contact. $L_{\mathcal{A}_{i}}+L_{\mathcal{B}_{i}}$ is positive definite (thus invertible) in the generic case where the $2^{\text {nd }}$ order approximations to the two surfaces maintains point contact.

\section{Equilibrium Postures}

Let $U(q)$ be a potential energy (e.g., the gravitational potential), that influences $\mathcal{B}$. A necessary and sufficient condition for $q_{0}$ to be an equilibrium configuration is that $\nabla U\left(q_{0}\right)$ lie in the normal cone $N\left(q_{0}\right)$ [8]:

$$
\lambda_{1} n_{1}\left(q_{0}\right)+\cdots+\lambda_{k} n_{k}\left(q_{0}\right)=\nabla U\left(q_{0}\right) \text { for } \lambda_{i} \geq 0 \text {. }
$$

The following definition and theorem provide the basis for our stability analysis.
Definition 1 The relative curvature form associated with $U$ at an equilibrium configuration $q_{0}$ is:

$\kappa v\left(q_{0}, \dot{q}\right)=\sum_{i=1}^{k} \lambda_{i} \kappa_{i}\left(q_{0}, \dot{q}\right)-\dot{q}^{T} D^{2} U\left(q_{0}\right) \dot{q} \quad$ for $\dot{q} \in T_{q_{0}} \mathcal{S}$

where $\kappa_{i}\left(q_{0}, \dot{q}\right)$ is the curvature of $\mathcal{S}_{i}, \mathcal{S}=\Pi_{i=1}^{k} \mathcal{S}_{i}$, and the $\lambda_{i}$ 's are the equilibrium-posture coefficients, The relative curvature form associated with the gravitational potential is called the gravity relative curvature form, and is denoted $\kappa_{G}\left(q_{0}, \dot{q}\right)$. For generic grasps with $k \leq 6$ contacts, the $\lambda_{i}$ are uniquely determined by Eq. (3).

Theorem 1 (Stability Test) Let $U$ be a potential energy function, and let $\mathcal{B}$ be at an equilibrium configuration $q_{0}$, contacted by $k$ bodies $\mathcal{A}_{1}, \ldots, \mathcal{A}_{k}$. Assume that the matrix of c-obstacle normals, $\left[n_{1} \cdots n_{k}\right]$, has full rank (this is the generic case). Then for $k \geq 6$ contacts, the equilibrium $q_{0}$ is locally stable if there is a subset of 6 linearly independent c-obstacle normals such that

$$
\nabla U\left(q_{0}\right) \in \text { interior }\left(N^{\prime}\left(q_{0}\right)\right)
$$

where $N^{\prime}\left(q_{0}\right)$ is the cone spanned by these normals. For $k<6$ contacts, the equilibrium is locally stable if first,

$$
\nabla U\left(q_{0}\right) \in \text { interior }\left(N\left(q_{0}\right)\right),
$$

where $N\left(q_{0}\right)$ is the normal cone at $q_{0}$, and second, if

$$
\kappa_{U}\left(q_{0}, \dot{q}\right)<0 \text { for all } \dot{q} \in T_{q_{0}} \mathcal{S},
$$

The proof, with some discussion of what occurs when $\nabla U$ lies on the boundary of $N\left(q_{0}\right)$, is given in $[1,8]$.

\section{The Equilibrium Regions}

We now restrict our attention to the case of a uniform gravitational field. We assume the contacts are held fixed, while the object's center of mass varies in space. This section establishes the procedure to determine, for a given configuration $q_{0}$, the region where the center of mass must lie for equilibrium. We denote this equilibrium region by $\mathcal{E}\left(q_{0}\right)$. The subsequent sections show how to determine the stable equilibrium region (i.e, the stable subsets of $\left.\mathcal{E}\left(q_{0}\right)\right)$ for one, two, three, and more contact points. These stable regions, denoted $\mathcal{E S}\left(q_{0}\right)$, are useful for planning quasi-static locomotion of articulated mechanisms on rough terrain.

In the following, the vector $\boldsymbol{r}_{\mathrm{cm}}$ is the location of $\mathcal{B}$ 's center of mass, expressed in $\mathcal{B}$ 's body coordinates. The world coordinates of $\boldsymbol{r}_{\mathrm{cm}}$ are given by $x_{\mathrm{cm}}(q)=$ $R(\theta) r_{\mathrm{cm}}+d$. Tangent vectors are pairs $\dot{q}=(v, \omega)$, where $v=\dot{d}$ is the linear velocity of $\mathcal{B}$ 's frame origin 
and $\omega=\dot{\theta}$ the angular velocity of $\mathcal{B}$. For simplicity, let $\rho_{\mathrm{cm}}(\theta)=\left(x_{\mathrm{cm}}, y_{\mathrm{cm}}, z_{\mathrm{cm}}\right)^{T}=R(\theta) r_{\mathrm{cm}}$.

The gravitational potential is $U(q)=m g\left(e \cdot x_{\mathrm{cm}}(q)\right)$, where $m$ is the mass of $\mathcal{B}, g$ is the gravity constant, and $e=(0,0,1)^{T}$ is the vertical direction. The gradient and Hessian matrix of $U(q)$ are:

$$
\nabla U(q)=m g\left(e, \rho_{\mathrm{cm}}(\theta) \times e\right)^{T}
$$

where $\rho_{\mathrm{cm}} \times e=\left(y_{\mathrm{cm}},-x_{\mathrm{cm}}, 0\right)^{T}$, and

$$
D^{2} U(q)=m g\left[\begin{array}{cc}
O_{3} & O_{3} \\
O_{3} & \left(\left[\rho_{\mathrm{cm}}(\theta) \times\right][e \times]\right)_{s}
\end{array}\right]
$$

where $O_{3}$ is a $3 \times 3$ matrix of zeroes, and

$$
\left(\left[\rho_{\mathrm{cm}}(\theta) \times\right][e \times]\right)_{s}=\left[\begin{array}{ccc}
-z_{\mathrm{cm}} & 0 & \frac{1}{2} x_{\mathrm{cm}} \\
0 & -z_{\mathrm{cm}} & \frac{1}{2} y_{\mathrm{cm}} \\
\frac{1}{2} x_{\mathrm{cm}} & \frac{1}{2} y_{\mathrm{cm}} & 0
\end{array}\right]
$$

Note that the height of $\mathcal{B}$ 's center of mass, $z_{\mathrm{cm}}$, appears in the expression for $D^{2} U\left(q_{0}\right)$ but not in $\nabla U\left(q_{0}\right)$. Hence $\mathcal{E}\left(q_{0}\right)$ can be parametrized by the variables $x_{\mathrm{cm}}$ and $y_{\mathrm{cm}}$. However, the stability of each equilibria is dependent on $z_{\mathrm{cm}}$. Due to the $z_{\mathrm{cm}}$-invariance of Eq. (8), all possible vectors $\nabla U(q)$ form a 2-dimensional affine plane in $T_{q} \mathcal{C}$. Let $L$ denote this plane and let $V$ be the subspace of translational velocities in $T_{q} \mathcal{C}$. It follows from (8) that $L$ is orthogonal to $V$. For equilibrium, the vector $\nabla U\left(q_{0}\right)$ must lie in the normal cone $N\left(q_{0}\right)$. Every point in $T_{q_{0}} \mathcal{C}$ where $L$ intersects $N\left(q_{0}\right)$ determines a value of $\left(x_{\mathrm{cm}}, y_{\mathrm{cm}}\right)$ which is a feasible equilibrium of $\mathcal{B}$. Every vertical line passing through $\left(x_{\mathrm{cm}}, y_{\mathrm{cm}}\right)$ belongs to $\mathcal{E}\left(q_{0}\right)$.

Since $N\left(q_{0}\right)$ is convex, the intersection of $L$ with $N\left(q_{0}\right)$, if non-empty, is convex and connected. If $L$ intersects $N\left(q_{0}\right)$ at a single point (this is the generic case for one to four contacts, if the intersection is non-empty), the equilibrium region $\mathcal{E}\left(q_{0}\right)$ consists of a single vertical line [8]. If the intersection is a line segment (generically the case for five contacts), $\mathcal{E}\left(q_{0}\right)$ consists of a vertical strip. Finally, $L$ may intersect $N\left(q_{0}\right)$ over a convex polygon (this occurs generically for six or more contacts), in which case $\mathcal{E}\left(q_{0}\right)$ is a vertical prism with polygonal cross-section.

Since $L$ is orthogonal to $V$, a necessary condition for $L$ to have a non-empty intersection with $N\left(q_{0}\right)$ is that the projections of $L$ and $N\left(q_{0}\right)$ onto $V$ intersect each other. The projection of $L$ is the point $e=(0,0,1)$. The projection of $N\left(q_{0}\right)$ is the positive span of $\hat{l}_{1}, \ldots, \hat{l}_{k}$. Hence $e$ must lie in this span for an equilibrium to exist. Further, since $\nabla U(q)$ always has zero torque component in the $z$ direction, for equilibrium the net torque generated by the reaction forces must also have a zero $z$ coordinate. These notions are summarized as follows.
Proposition 5.1 Let $\mathcal{B}$ be in frictionless contact with $k$ stationary bodies under the influence of gravity. Then the equilibrium region of $\mathcal{B}$, denoted $\mathcal{E}$, is non-empty iff (a) the normals $\hat{l}_{1}, \ldots, \hat{l_{k}}$ positively span $e=(0,0,1)$,

$$
e=\frac{1}{m g} \sum_{i=1}^{k} \lambda_{i} \hat{l}_{i} \text { for some } \lambda_{i} \geq 0 \text {. }
$$

and (b) for those values of $\lambda_{i}$ the net torque due to the reaction forces has a vanishing $z$ coordinate,

$$
e \cdot \sum_{i=1}^{k} \lambda_{i}\left(\rho_{i} \times \hat{l}_{i}\right)=0
$$

We now examine poses with one, two, or three contacts. In each of the following sections, we first determine $\mathcal{E}\left(q_{0}\right)$. Then we determine the stable subset via the gravity relative-curvature form. The evaluation of this form requires us to find a basis for $T_{q_{0}} \mathcal{S}$.

\section{Stable One-Contact Postures}

According to Prop. 5.1, the contact normal $\hat{l}_{1}$ of a singlecontact equilibrium must point vertically, with $\lambda_{1}=m g$. Then $\mathcal{E}\left(q_{0}\right)$ consists of the vertical line passing through the contact point. The stable subset of this line can be found as follows. Let $s_{\mathrm{cm}}$ be the vertical height of $\mathcal{B}$ 's center of mass (located on $l_{1}$ ) from the contact. $s_{\mathrm{cm}}$ is positive (negative) when the center of mass is above (below) the contact point. Locate the origin of $\mathcal{B}$ 's body frame on $l_{1}$, at a fixed distance $\rho_{1}$ from the contact, such that $\rho_{1}$ is positive (negative) when $\mathcal{B}$ 's origin lies above (below) the contact. In these coordinates, $\rho_{\mathrm{cm}}=$ $\left(x_{\mathrm{cm}}, y_{\mathrm{cm}}, z_{\mathrm{cm}}\right)=\left(0,0,-\rho_{1}+s_{\mathrm{cm}}\right)$. Formula (9) yields:

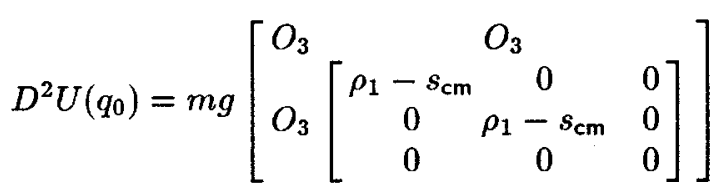

In order to determine $\mathcal{E S}\left(q_{0}\right)$, we must evaluate $\kappa_{G}\left(q_{0}, \dot{q}\right)$ on the 5-dimensional tangent space $T_{q_{0}} \mathcal{S}_{1}$. When $\mathcal{B}$ 's frame origin is on $l_{1}$, the normal vector to $\mathcal{S}_{1}$ at $q_{0} \in \mathcal{S}_{1}$ is $n_{1}\left(q_{0}\right)=\left(\hat{l}_{1}, 0\right)$. Hence the tangent vectors $\dot{q}=(v, \omega) \in$ $T_{q_{0}} \mathcal{S}_{1}$ satisfy $\left(\hat{l}_{1}, 0\right) \cdot(v, \omega)=\hat{l}_{1} \cdot v=0$. The tangent vector $\dot{q}=\left(0, \hat{l}_{1}\right) \in T_{q_{0}} \mathcal{S}_{1}$, corresponding to rotation of $\mathcal{B}$ about the contact normal $\hat{l}_{1}$, is an eigenvector with zero eigenvalue both of the curvature form $\kappa_{1}\left(q_{0}, \dot{q}\right)$ and of $D^{2} U\left(q_{0}\right)$, and thereof of $\kappa_{G}\left(q_{0}, \dot{q}\right)$. Thus, $\kappa_{G}\left(q_{0}, \dot{q}\right)$ can be at most negative semi-definite on $T_{q_{0}} \mathcal{S}_{1}$. To determine the values of $s_{\mathrm{cm}}$ which make the remaining eigenvalues negative, it suffices to evaluate $\kappa_{G}\left(q_{0}, \dot{q}\right)$ on the subspace of $T_{q_{0}} \mathcal{S}_{1}$ orthogonal to the vector $\left(0, \hat{l}_{1}\right)$. We denote this subspace by $W$. Let $G$ be the restriction of the $6 \times 6$ matrix $D n_{1}\left(q_{0}\right)-D^{2} U\left(q_{0}\right)$ to $W$. 
To find an expression for $\kappa_{1}\left(q_{0}, \dot{q}\right)$ we use Lemma 3.1, which requires expressions for $L_{\mathcal{A}_{1}}$ and $L_{\mathcal{B}_{1}}$. Define the principal axes of curvature of $\mathcal{B}$ at the contact as basis vectors for a contact reference frame. Let $k_{x}^{b}$ and $k_{y}^{b}$ be the principal curvatures of $\mathcal{B}$ at the contact. Then the matrix representation of $L_{\mathcal{B}_{1}}$ is $B_{1}=\left[\begin{array}{cc}k_{x}^{b} & 0 \\ 0 & k_{y}^{b}\end{array}\right]$.

Let $k_{x}^{a}$ and $k_{y}^{a}$ be the principal curvatures of $\mathcal{A}_{1}$ at the contact, and let $\theta$ be the (fixed) angle between the principal axes of curvature of $\mathcal{A}_{1}$ and $\mathcal{B}$ at the contact. The matrix representation of $L_{\mathcal{A}_{1}}$ is:

$$
A_{1}=\left[\begin{array}{cc}
k_{x}^{a} \mathrm{c}^{2} \theta+k_{y}^{a} \mathrm{~s}^{2} \theta & \left(k_{x}^{a}-k_{y}^{a}\right) \mathrm{c}(\theta) \mathrm{s}(\theta) \\
\left(k_{x}^{a}-k_{y}^{a}\right) \mathrm{c}(\theta) \mathrm{s}(\theta) & k_{x}^{a} \mathrm{~s}^{2} \theta+k_{y}^{a} \mathrm{c}^{2} \theta
\end{array}\right]
$$

where $c(\theta)=\cos (\theta)$ and $s(\theta)=\sin (\theta)$.

The formula for $\kappa_{1}\left(q_{0}, \dot{q}\right)$ simplifies considerably when we locate $\mathcal{B}$ 's frame origin at a distance $\rho_{1}=1 / k_{y}^{b}$ on $l_{1}$. (If $k_{y}^{b}=0$, a similar computation holds with $\rho_{1}=1 / k_{x}^{b}$.) Using formula (13) for $D^{2} U\left(q_{0}\right)$, we find:

$$
\begin{aligned}
G & =m g\left(\frac{1}{\Delta}\left[\begin{array}{cccc}
K_{11} & K_{12} & K_{13} & 0 \\
K_{12} & K_{22} & K_{23} & 0 \\
K_{13} & K_{23} & K_{33} & 0 \\
0 & 0 & 0 & 0
\end{array}\right]\right. \\
- & {\left.\left[\begin{array}{ccccc}
0 & 0 & 0 & 0 \\
0 & 0 & 0 & 0 \\
0 & 0 & \frac{1}{k_{y}^{b}}-s_{\mathrm{cm}} & 0 \\
0 & 0 & 0 & \frac{1}{k_{y}^{b}}-s_{\mathrm{cm}}
\end{array}\right]\right) }
\end{aligned}
$$

where $\Delta=\operatorname{det}\left(A_{1}+B_{1}\right)>0$ for a point contact.

$$
\begin{aligned}
\Delta= & k_{x}^{a} k_{y}^{a}+k_{x}^{b} k_{y}^{b}+\left(k_{y}^{a} k_{x}^{b}+k_{x}^{a} k_{y}^{b}\right) \cos ^{2}(\theta) \\
& +\left(k_{x}^{a} k_{x}^{b}+k_{y}^{a} k_{y}^{b}\right) \sin ^{2}(\theta)
\end{aligned}
$$

The expressions for the other terms in (14) are:

$$
\begin{aligned}
& K_{11}=k_{x}^{b}\left(k_{x}^{a} k_{y}^{a}+k_{x}^{a} k_{y}^{b} \cos ^{2}(\theta)+k_{y}^{a} k_{y}^{b} \sin ^{2}(\theta)\right) \\
& K_{22}=k_{y}^{b}\left(k_{x}^{a} k_{y}^{a}+k_{y}^{a} k_{x}^{b} \cos ^{2}(\theta)+k_{x}^{a} k_{x}^{b} \sin ^{2}(\theta)\right) \\
& K_{33}=\left(k_{x}^{b}-k_{y}^{b}\right)\left(k_{x}^{a}+k_{y}^{b}\right)\left(k_{y}^{a}+k_{y}^{b}\right) / k_{y}^{b} \\
& K_{12}=\left(k_{x}^{a}-k_{y}^{a}\right) k_{x}^{b} k_{y}^{b} \cos (\theta) \sin (\theta) \\
& K_{13}=\frac{k_{x}^{b}-k_{y}^{b}}{k_{y}^{b}}\left(k_{x}^{a} k_{y}^{a}+k_{x}^{a} k_{y}^{b} \mathbf{c}^{2}(\theta)+k_{y}^{a} k_{y}^{b} \mathbf{s}^{2}(\theta)\right) \\
& K_{23}=\left(k_{x}^{a}-k_{y}^{a}\right)\left(k_{x}^{b}-k_{y}^{b}\right) \cos (\theta) \sin (\theta) .
\end{aligned}
$$

We call a surface convex if its principal curvatures are both positive, concave if they are both negative, and a saddle if they are of mixed sign. The next proposition generalizes Kriegman's result for an object resting on a flat support [5], to any support.

\begin{tabular}{|c|c|c|l|}
\hline & Object & Support & Stability \\
\hline \hline 1 & concave & concave & \\
2 & concave & saddle & case not possible \\
3 & saddle & concave & \\
\hline 4 & convex & convex & \\
5 & convex & saddle & always unstable \\
6 & saddle & convex & \\
7 & flat & convex & \\
\hline 8 & convex & concave & stable iff \\
9 & concave & convex & $s_{\mathrm{cm}}<\min \left(1 / k_{x}^{b}, 1 / k_{y}^{b}\right)$ \\
10 & saddle & saddle & \\
\hline 11 & convex & flat & neutrally stable under \\
& & & pure translation; \\
& & & otherwise stable iff \\
& & & $s_{\mathrm{cm}}<\min \left(1 / k_{x}^{b}, 1 / k_{y}^{b}\right)$ \\
\hline
\end{tabular}

Table 1: Stability conditions for a single-contact pose

Proposition 6.1 Let $\mathcal{B}$ be a $3 D$ object resting in static frictionless equilibrium on a single support, whose contact line is $l_{1}$. The stability region is the lower halfline of $l_{1}$ below the minimum radius of curvature of $\mathcal{B}$ at the contact point when: one of the contacting surfaces is convex and the other is concave; both surfaces are saddles; and the object is convex and the support is flat. The equilibria of all other physically realizable cases are unstable. See Table 1.

Proof: For cases 1,2,3 in Table 1, the two bodies must interpenetrate; these cases are physically unrealizable. In cases $4,5,6$ there exists a vertical plane passing through the contact point along which the sectional curvature of each body is positive. But for planar bodies, if object and support are both convex at the contact, the equilibrium is unstable $[1,8]$. Case 7 can be derived using a similar argument.

Now consider cases 8,9 , and 10 . It is evident from stability results for planar bodies $[1,8]$ that the condition $s_{\mathrm{cm}}<\min \left(1 / k_{x}^{b}, 1 / k_{y}^{b}\right)$ is necessary for stability. We wish to show this condition is also sufficient. For stability, $G$ must be negative definite. By considering its principal minors, we find that $G<0$ iff:

$$
\begin{gathered}
k_{x}^{a} k_{x}^{b}\left(k_{y}^{a}+k_{y}^{b}\right) \mathrm{c}^{2}(\theta)+k_{y}^{a} k_{x}^{b}\left(k_{x}^{a}+k_{y}^{b}\right) \mathrm{s}^{2}(\theta)<0 \\
k_{x}^{a} k_{y}^{a} k_{x}^{b} k_{y}^{b}>0 \\
k_{x}^{a} k_{y}^{a} k_{y}^{b}\left(-1+k_{x}^{b} s_{\mathrm{cm}}\right)<0 \\
s_{\mathrm{cm}}-1 / k_{y}^{b}<0
\end{gathered}
$$

Without loss of generality, assume $k_{y}^{b} \geq k_{x}^{b}$ and $k_{y}^{a} \geq k_{x}^{a}$. Then it can be established that $\left(k_{y}^{a}+k_{x}^{b}\right),\left(k_{x}^{a}+k_{y}^{b}\right)$, and $\left(k_{y}^{a}+k_{y}^{b}\right)$ are all positive. To see this, consider 


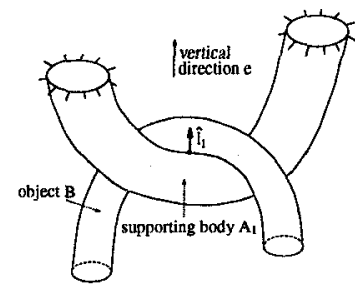

Figure 1: An example of saddle/saddle contact

the test vectors $v_{1}=(1,0), v_{2}=(\mathrm{c}(\theta), \mathrm{s}(\theta))$, and $v_{3}=(-\mathrm{s}(\theta), \mathrm{c}(\theta))$. Since $A_{1}+B_{1}$ is generically positive definite, $v_{i}^{T}\left[A_{1}+B_{1}\right] v_{i}>0$ for $i=1,2,3$. This gives the following three inequalities: $k_{x}^{b}+k_{x}^{a} \mathrm{c}^{2}(\theta)+k_{y}^{a} \mathrm{c}^{2}(\theta)>0$, $k_{x}^{a}+k_{x}^{b} \mathrm{c}^{2}(\theta)+k_{y}^{b} \mathrm{~s}^{2}(\theta)^{2}>0$, and $k_{y}^{a}+k_{x}^{b} \mathrm{~s}^{2}(\theta)+k_{y}^{b} \mathrm{c}^{2}(\theta)>$ 0 . Substituting $k_{y}^{b} \geq k_{x}^{b}$ and $k_{y}^{a} \geq k_{x}^{a}$ gives the result.

In case $8, \mathcal{B}$ is convex and $\mathcal{A}$ is concave, so that $k_{x}^{b}, k_{y}^{b}>0$ while $k_{x}^{a}, k_{y}^{a}<0$. In case $9 \mathcal{B}$ is concave and $\mathcal{A}$ is convex, so that $k_{x}^{b}, k_{y}^{b}<0$, while $k_{x}^{a}, k_{y}^{a}>0$. In both cases $k_{x}^{a} k_{x}^{b}$ and $k_{y}^{a} k_{x}^{b}$ are negative, and $\left(k_{y}^{a}+k_{y}^{b}\right)$ and $\left(k_{x}^{a}+k_{y}^{b}\right)$ are positive. Hence condition (16) is satisfied. Condition (17) is also satisfied. Condition (18) is satisfied iff $s_{\mathrm{cm}}<1 / k_{x}^{b}$. Condition (19) is satisfied iff $s_{\mathrm{cm}}<1 / k_{y}^{b}$. So $s_{\mathrm{cm}}<\min \left(1 / k_{x}^{b}, 1 / k_{y}^{b}\right)$ implies stability.

In case 10 both surfaces are saddles at the contact. $k_{x}^{a}, k_{x}^{b}<0, k_{y}^{a}, k_{y}^{b}>0$,so (17) is satisfied. The requirement $\Delta>0$ can be written as $\tan ^{2}(\theta)>$ $-\frac{\left(k_{x}^{a}+k_{x}^{b}\right)\left(k_{y}^{a}+k_{y}^{b}\right)}{\left(k_{y}^{a}+k_{x}^{b}\right)\left(k_{x}^{a}+k_{y}^{b}\right)}$. But $\left(k_{y}^{a}+k_{x}^{b}\right)>0$, so $-\frac{\left(k_{x}^{a}+k_{y}^{b}\right)}{\left(k_{y}^{a}+k_{x}^{b}\right)}>$ $-\frac{k_{x}^{a}}{k_{y}^{a}}$, and therefore $\tan ^{2}(\theta)>-\frac{k_{x}^{a}\left(k_{y}^{a}+k_{y}^{b}\right)}{k_{y}^{a}\left(k_{x}^{a}+k_{y}^{b}\right)}$, which is condition (16). Condition (18) is satisfied iff $s_{\mathrm{cm}}<1 / k_{x}^{b}$, and condition (19) is satisfied iff $s_{\mathbf{c m}}<1 / k_{y}^{b}$, giving the table's result. The result for case 11 can be derived by setting $k_{x}^{a}=k_{y}^{a}=0$ in $G$.

\section{Stable Two-Contact Postures}

First we determine the two-contact equilibrium region of $\mathcal{B}$. Prop. 5.1 implies that for equilibrium, the contact normals $\hat{l}_{1}$ and $\hat{l}_{2}$ must positively span the vertical direction $e$, and furthermore that the contact lines must lie in the same vertical plane, denoted $\mathcal{P}$.

\section{The Contact Lines Intersect at a Point}

If $l_{1}$ and $l_{2}$ intersect at some point $p \in \mathcal{P}, \mathcal{E}\left(q_{0}\right)$ is the vertical line passing through $p$, denoted $l^{\prime}$. To determine the stable subset of $l^{\prime}$, we must evaluate $\kappa_{G}\left(q_{0}, \dot{q}\right)$ on the 4-dimensional tangent space $T_{q_{0}} \mathcal{S}$, where $\mathcal{S}=\mathcal{S}_{1} \cap \mathcal{S}_{2}$. Suppose the object's frame origin is located at $p$. Let $v$ be the unit vector perpendicular to $\mathcal{P}$. Consider the four vectors in the ambient tangent space $T_{q_{0}} \mathbb{R}^{6}: u_{1}=(v, \overrightarrow{0})$, $u_{2}=(\overrightarrow{0},(0,0,1)), u_{3}=(\overrightarrow{0},(0,1,0)), u_{4}=(\overrightarrow{0},(1,0,0))$, where $\overrightarrow{0}=(0,0,0)$. The first vector corresponds to translation in the horizontal direction $v$, and the other three correspond to rotation of $\mathcal{B}$ about three orthogonal axes through the point $p$. All four vectors are orthogonal to $n_{1}\left(q_{0}\right)=\left(\hat{l}_{1}, 0\right)$ and $n_{2}\left(q_{0}\right)=\left(\hat{l}_{2}, 0\right)$, and they are linearly independent. Hence they form a basis for $T_{q_{0}} \mathcal{S}$. In the following, $B$ is the $6 \times 4$ matrix $\left(\begin{array}{llll}u_{1} & u_{2} & u_{3} & u_{4}\end{array}\right)$.

Recall that $\kappa_{G}\left(q_{0}, \dot{q}\right)=\sum_{i=1}^{2} \lambda_{i} \kappa_{i}\left(q_{0}, \dot{q}\right)-\dot{q}^{T} D^{2} U\left(q_{0}\right) \dot{q}$. Let $\alpha_{i}$ be the absolute angle between $\hat{l}_{i}$ and $e$. A little algebra reveals

$$
\lambda_{1}=\frac{m g \sin \left(\alpha_{2}\right)}{\sin \left(\alpha_{1}+\alpha_{2}\right)} \quad \text { and } \quad \lambda_{2}=\frac{m g \sin \left(\alpha_{1}\right)}{\sin \left(\alpha_{1}+\alpha_{2}\right)},
$$

where $\sin \left(\alpha_{1}\right), \sin \left(\alpha_{2}\right)$, and $\sin \left(\alpha_{1}+\alpha_{2}\right)$ are all positive. The center of mass lies on the vertical line $l^{\prime}$, so $\rho_{\mathrm{cm}}=$ $\left(0,0, z_{\mathrm{cm}}\right)^{T}$. The restriction of $D^{2} U\left(q_{0}\right)$ to $T_{q_{0}} \mathcal{S}$ is:

$$
B^{T} D^{2} U\left(q_{0}\right) B=m g\left[\begin{array}{cc}
O_{2} & O_{2} \\
O_{2} & -z_{\mathrm{cm}} I_{2}
\end{array}\right]
$$

where $I_{2}$ is the $2 \times 2$ identity. Consider Eq. (2) for $\kappa_{i}\left(q_{0}, \dot{q}\right)$. Since the object frame origin lies at $p, \rho_{i}$ can be written as $\rho_{i}=-\rho_{i} \hat{l}_{i}$, where $\rho_{i}$ is the signed distance from $p$ to the $i^{\text {th }}$ contact point, positive when $p$ is on $\mathcal{B}$ 's side of the contact and negative when $p$ is on $\mathcal{A}_{i}$ 's side. It also follows that $c_{i}=\sqrt{1+\left\|\rho_{i} \times \hat{l}_{i}\right\|^{2}}$ is unity.

Let $G$ be the $4 \times 4$ matrix representing the restriction of the matrix associated with $\kappa_{G}\left(q_{0}, \dot{q}\right)$ to $T_{q_{0}} \mathcal{S}$.

$$
\begin{gathered}
G=\sum_{i=1}^{2} \lambda_{i} B^{T}\left\{\left[\begin{array}{cc}
I & \rho_{i}\left[\hat{l}_{i} \times\right] \\
0 & {\left[\hat{l}_{i} \times\right]}
\end{array}\right]^{T}\right. \\
{\left[\begin{array}{cc}
L_{\mathcal{B}_{i}} L_{r e l}^{-1} L_{\mathcal{A}_{i}} & -L_{\mathcal{A}_{i}} L_{r e l}^{-1} \\
-L_{r e l}^{-1} L_{\mathcal{A}_{i}} & -L_{r e l}^{-1}
\end{array}\right]\left[\begin{array}{cc}
I & \rho_{i}\left[\hat{l}_{i} \times\right] \\
0 & {\left[\hat{l}_{i} \times\right]}
\end{array}\right]} \\
\left.+\rho_{i}\left[\begin{array}{cc}
0 & 0 \\
0 & {\left[\hat{l}_{i} \times\right]^{T}\left[\hat{l}_{i} \times\right]}
\end{array}\right]\right\} B+z_{\mathrm{cm}}\left[\begin{array}{cc}
O_{2} & O_{2} \\
O_{2} & I_{2}
\end{array}\right]
\end{gathered}
$$

where $L_{\text {rel }}=L_{\mathcal{A}_{i}}+L_{\mathcal{B}_{i}}$. The restriction of $G$ to the subspace spanned by the vectors $u_{1}=(v, \overrightarrow{0})$ and $u_{2}=$ $(\overrightarrow{0}, e)$, denoted $\tilde{G}$, is unaffected by $z_{\mathrm{cm}}$. So a necessary condition for the existence of stable equilibria is that $\tilde{G}$ be negative definite. This condition is also sufficient, since away from this subspace $G$ is negative definite for $z_{\mathrm{cm}}<<0$. Since $z_{\mathrm{cm}}$ appears linearly in the diagonals of $G$, if $\mathcal{E S}\left(q_{0}\right)$ is non-empty, it contains a lower halfline of $l^{\prime}$. In fact, if non-empty $\mathcal{E S}\left(q_{0}\right)$ will be exactly a lower half-line of $l^{\prime}$, because by varying $z_{\mathrm{cm}}$ we vary $G$ along a line in the space of symmetric matrices, and negative-definite matrices are a convex set in this space. We compute the stable values of $z_{\mathrm{cm}}$ by writing $G<0$ in terms of the principal minors of $G$; $z_{\mathrm{cm}}$ will appear linearly in the $3 \times 3$ minor and quadratically in the $4 \times$ 4 minor. Hence there is a closed-form solution for the point where the stable half-line begins. 


\section{Contact Lines are Parallel/Antiparallel}

If $l_{1}$ and $l_{2}$ are parallel, $\hat{l}_{1}=\hat{l}_{2}=e, \mathcal{E}\left(q_{0}\right)$ is the vertical strip in $\mathcal{P}$ bounded by $l_{1}$ and $l_{2}$. If $l_{1}$ and $l_{2}$ are antiparallel (without loss of generality, suppose $\hat{l}_{1}=e$ and $\left.\hat{l}_{2}=-e\right), \mathcal{E}\left(q_{0}\right)$ is the half-plane of $\mathcal{P}$ bounded by $l_{1}$ and not containing $l_{2}$. In either case, given $v$, the unit vector perpendicular to $\mathcal{P}$, consider the four twists $u_{1}=(v \times e, \overrightarrow{0}), u_{2}=(v, \overrightarrow{0}), u_{3}=(\overrightarrow{0}, v \times e), u_{4}=(\overrightarrow{0}, e)$. The first two are instantaneous horizontal translations of $\mathcal{B}$, while the third and fourth are instantaneous rotation of $\mathcal{B}$ about the axes $v \times e$ and $e$. These four twists form a basis for $T_{q_{0}} \mathcal{S}$. Let $B=\left(u_{1} u_{2} u_{3} u_{4}\right)$.

Suppose that the $x$-axis of the world frame is aligned horizontally with $\mathcal{P}$. Denote the $x$-coordinates of $\rho_{1}$ and $\rho_{2}$ (the vectors from the origin of the body-frame to the contact points, in world coordinates) as $\rho_{1 x}$ and $\rho_{2 x}$ respectively. From (3), we find that in either case,

$$
\frac{\lambda_{1}}{c_{1}}=\frac{m g\left|\rho_{2 x}-x_{\mathrm{cm}}\right|}{\left|\rho_{1 x}-\rho_{2 x}\right|} \text { and } \frac{\lambda_{2}}{c_{2}}=\frac{m g\left|\rho_{1 x}-x_{\mathrm{cm}}\right|}{\left|\rho_{1 x}-\rho_{2 x}\right|}
$$

With the chosen frames, $\rho_{\mathrm{cm}}=\left(x_{\mathrm{cm}}, 0, z_{\mathrm{cm}}\right)$. Therefore the restriction of $D^{2} U\left(q_{0}\right)$ to $T_{q_{0}} \mathcal{S}$ is:

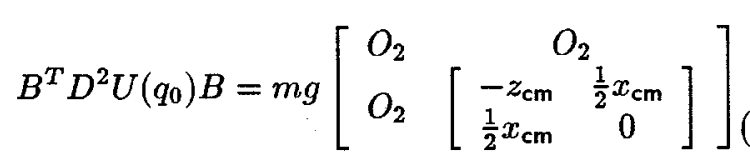

Now we have all the terms required to develop a symbolic expression for $G$ like that in (21). The four minors of $G$ yield four symbolic inequalities that must be satisfied for $G<0$. Three of these inequalities place restrictions on $x_{\mathrm{cm}}$ (so that $\mathcal{E S}\left(q_{0}\right)$ is contained in a vertical substrip of $\left.\mathcal{E}\left(q_{0}\right)\right)$. The remaining inequality creates a upper bound on $z_{\mathrm{cm}}$ which is a ratio of polynomials in $x_{\mathrm{cm}}$.

\section{Stable Three-Contact Postures}

Assume $\mathcal{B}$ is now supported by three contacts. For equilibrium the contact normals must positively span the vertical direction $e$. Also, the three contacts must exert zero net moment around any vertical axis. Hence, there must generically exist a vertical line, $\mathcal{V}$, that intersects all three contact lines $l_{1}, l_{2}, l_{3}$. (We postpone treatment of certain degenerate cases, as when one or more of the contact lines are vertical, or their projections onto the horizontal plane are parallel.) We place the object frame origin on $\mathcal{V}$ with its $z$-axis vertical. Let $z_{i} e$ be the vector from the frame origin to the intersection of $l_{i}$ with $\mathcal{V}$. Let $\left(\hat{l}_{i}\right)_{x y}=\left(\hat{l}_{i x}, \hat{l}_{i y}\right)$ be the projection of $\hat{l}_{i} \in T R^{3}$ onto the horizontal $x y$-plane. Let $\left(n_{i}\right)_{x y}=\left(\left(n_{i}\right)_{x},\left(n_{i}\right)_{y}\right)$ be the projection of $n_{i} \in T_{q 0} \mathcal{C}$ onto the horizontal $x y$-plane. Note that $c_{i}\left(n_{i}\right)_{x y}=\left(\hat{l}_{i}\right)_{x y}$. Let $\psi_{i}$ be the angle between $\hat{l}_{i}$ and the vertical. Let $\theta_{i}$ be the angle between $\left(\hat{l}_{i}\right)_{x y}$ and the x-axis. See Fig. 2.

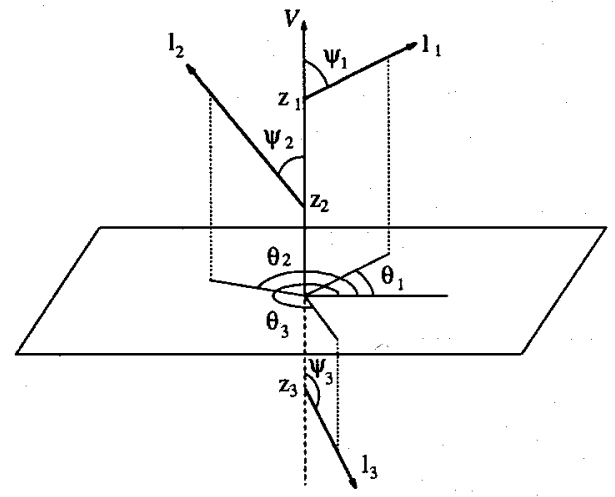

Figure 2: $l_{1}, l_{2}, l_{3}$ intersect a common vertical line.

Lemma 8.1 An object generically supported by three contacts will be in equilibrium if and only if $e$ lies in the positive span of the contact directions $\hat{l}_{i}$ and

$$
\left(\begin{array}{l}
x_{\mathrm{cm}} \\
y_{\mathrm{cm}}
\end{array}\right)=-\sum_{i=1}^{3} \frac{\lambda_{i} z_{i}}{m g}\left(n_{i}\right)_{x y}=-\sum_{i=1}^{3} \frac{\lambda_{i} z_{i}}{m g c_{i}}\left(\hat{l}_{i}\right)_{x y}
$$

Proof: This follows trivially from the condition $\sum_{i=1}^{3} \lambda_{i}\left(\left(n_{i}\right)_{x},\left(n_{i}\right)_{y},\left(n_{i}\right)_{z},-z_{i}\left(n_{i}\right)_{y}, z_{i}\left(n_{i}\right)_{x}, 0\right)^{T}=$ $m g\left(0,0,1, y_{\mathrm{cm}},-x_{\mathrm{cm}}, 0\right)^{T}$.

Note that $c_{i}=\sqrt{1+z_{i}^{2} \mathrm{~s}^{2}\left(\psi_{\mathrm{i}}\right)}$. An algebraic solution for the $\lambda_{i}$ in terms of the quantities in Fig. 2 is

$$
\begin{aligned}
& \lambda_{1} / c_{1}=m g s\left(\psi_{2}\right) \mathrm{s}\left(\psi_{3}\right) \mathrm{s}\left(\theta_{2}-\theta_{3}\right) / \delta \\
& \lambda_{2} / c_{2}=m g s\left(\psi_{1}\right) \mathrm{s}\left(\psi_{3}\right) \mathrm{s}\left(\theta_{3}-\theta_{1}\right) / \delta \\
& \lambda_{3} / c_{3}=m g s\left(\psi_{1}\right) \mathrm{s}\left(\psi_{2}\right) \mathrm{s}\left(\theta_{1}-\theta_{2}\right) / \delta
\end{aligned}
$$

where $\delta=\mathrm{c}\left(\psi_{1}\right) \mathrm{s}\left(\psi_{2}\right) \mathrm{s}\left(\psi_{3}\right) \mathrm{s}\left(\theta_{2}-\theta_{3}\right)+$ $\mathrm{s}\left(\psi_{1}\right) \mathrm{c}\left(\psi_{2}\right) \mathrm{s}\left(\psi_{3}\right) \mathrm{s}\left(\theta_{3}-\theta_{1}\right)+\mathrm{s}\left(\psi_{1}\right) \mathrm{s}\left(\psi_{2}\right) \mathrm{c}\left(\psi_{3}\right) \mathrm{s}\left(\theta_{1}-\theta_{2}\right)$. Suppose that the center of mass coordinates satisfy Eq. (24). We now find a basis for the 3-dimensional space $T_{q_{0}} S$ such that each basis element is a pure instantaneous rotation (it is not necessary to choose such bases, but by doing so we aid visualization of the problem). The screw axis of any rotational twist basis element must intersect all of the $l_{i}$. The line $\mathcal{V}$ is one such axis. Three more can be found as follows. In the generic case, none of the $l_{i}$ are parallel. Select any one of the $l_{i}$, say $l_{1}$. The projections of $l_{2}$ and $l_{3}$ onto a plane normal to $l_{1}$ must intersect in at least one point. This means there is a line parallel to $l_{1}$ (that is, it intersects $l_{1}$ at infinity) which intersects $l_{2}$ and $l_{3}$. By permuting the lines in this argument, we discover axes parallel to each of the $l_{i}$ which intersect all the other $l_{j}$. Clearly, rotation about any one of these four axes (including $\mathcal{V}$ ) is a first-order free motion. In the generic case, any three of the rotations are linearly independent and form a basis for $T_{q_{0}} S$. Straightforward calculations establish the following result [8]. 
Lemma 8.2 In a generic three-contact equilibrium, there are four pure rotations in $T_{q_{0}} S$ with the following twist coordinates:

$$
\begin{aligned}
& b_{1}=\left(\left[\left(\lambda_{2} / c_{2} m g\right)\left(z_{3}-z_{2}\right) \hat{l_{2}}+z_{2} e\right] \times \hat{l_{1}}, \hat{l_{1}}\right)^{T} \\
& b_{2}=\left(\left[\left(\lambda_{1} / c_{1} m g\right)\left(z_{3}-z_{1}\right) \hat{l_{1}}+z_{1} e\right] \times \hat{l_{2}}, \hat{l_{2}}\right)^{T} \\
& b_{3}=\left(\left[\left(\lambda_{1} / c_{1} m g\right)\left(z_{2}-z_{1}\right) \hat{l_{1}}+z_{1} e\right] \times \hat{l_{3}}, \hat{l_{3}}\right)^{T} \\
& b_{4}=\left(\begin{array}{llllll}
0 & 0 & 0, & 0 & 0 & 1
\end{array}\right)^{T}
\end{aligned}
$$

We select a linearly independent set of these twists (say, $b_{1}, b_{2}$, and $\left.b_{3}\right)$ as a basis for $T_{q_{0}} S$. In this basis,

$$
G=\left(b_{1} b_{2} b_{3}\right)^{T}\left\{-D^{2} U+\sum_{i=1}^{3} \lambda_{i} D n_{i}\right\}\left(b_{1} b_{2} b_{3}\right)
$$

The eigenvalues of this 3-by-3 matrix can be written in closed form; the equilibrium is stable if all are negative.

For non-generic contact geometries, $\mathcal{E}\left(q_{0}\right)$ may not be a single vertical line. As a well-known example, if the contact normals all point vertically upward, $\mathcal{E}\left(q_{0}\right)$ is the vertical prism with edges $l_{1}, l_{2}, l_{3}$. If the supports are flat, as for an object resting on a flat plane, then the equilibria in this case are all neutrally stable (the proof is simple and given in [8]). An example with more complicated surface curvature is illustrated in Figure 4.

\section{A Computational Method}

For large numbers of contacts, it can be difficult to find a tractable symbolic expression that captures the stability of a pose without making some simplifying assumptions. This section summarizes a generally applicable numerical procedure to find $\mathcal{E}\left(q_{0}\right)$ and $\mathcal{E S}\left(q_{0}\right)$.

\subsection{Computing Equilibrium Regions}

With $k$ contacts, we may rewrite the equilibrium condition $\sum_{i=1}^{k} \lambda_{i} n_{i}=\nabla U$ in the following matrix form:

$$
\Upsilon\left(\lambda_{1} \cdots \lambda_{k} x_{\mathrm{cm}} y_{\mathrm{cm}}\right)^{T}=(0,0, m g, 0,0,0)^{T}
$$

where the matrix $\Upsilon$ is defined as

$\Upsilon=\left(n_{1}, \cdots, n_{k},(0,0,0,0, m g, 0)^{T},(0,0,0,-m g, 0,0)^{T}\right)$

Let $a$ be the dimension of Null( $\Upsilon$ ). Generically, we expect $a=2$ for $k=6, a=1$ for $k=5$, and $a=0$ for $k \leq 4$, but $a$ may be higher in various non-generic cases. However, by inspection there must be at least three linearly independent columns in $\Upsilon$, so $a<k$ in all cases. Supposing an equilibrium solution exists, we may write

$$
\left(\lambda_{1} \cdots \lambda_{k} x_{\mathrm{cm}} y_{\mathrm{cm}}\right)^{T}=\Upsilon^{\dagger}(0,0, m g, 0,0,0)^{T}+A \eta
$$

where $\Upsilon^{\dagger}$ is a right-inverse of $\Upsilon$, the columns of matrix $A$ form a basis for $\operatorname{Null}(\Upsilon)$, and $\eta \in \mathbb{R}^{a}$.
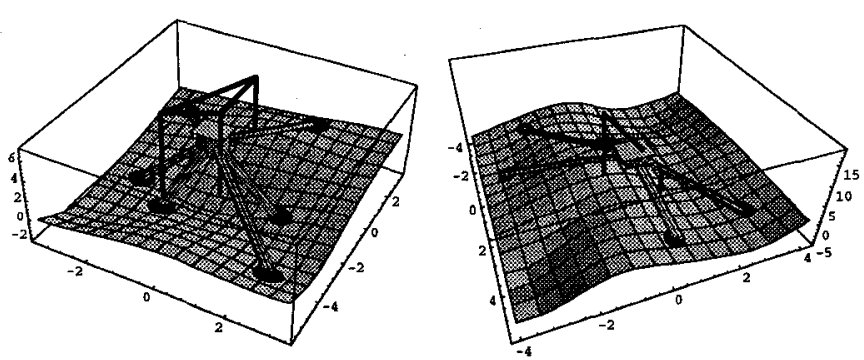

Figure 3: For 6 supports, $\mathcal{E S}\left(q_{0}\right)$ is generically an infinite vertical prism. For 5 supports $\mathcal{E S}\left(q_{0}\right)$ is generically a subset of a vertical strip, with a linear upper boundary.

If $a=0$, then there is only a single solution to check: if $\lambda_{i} \geq 0 \forall i$, then $\mathcal{E}\left(q_{0}\right)$ is the vertical line with the horizontal coordinates $x_{\mathrm{cm}}, y_{\mathrm{cm}}$.

If $a>0$, the set of equilibrium $\eta$ is an intersection of $k$ half-spaces determined by the inequalities $\lambda_{i} \geq 0$, and therefore, if non-empty, is a convex polyhedron $\mathcal{H}$ in $\mathbb{R}^{a}$. A point within $\mathcal{H}$ can be found using linear programming, then finding $\mathcal{H}$ is equivalent to finding the convex hull of $k$ points dual to the $k$ half-spaces. See, e.g. [10]. However, in our envisioned applications, $k$ and $a$ are small enough that it makes sense to find the vertices of $\mathcal{H}$ using a trivial method. Once we have found $\mathcal{H}$, we can map it to a region of $\left(x_{\mathrm{cm}}, y_{\mathrm{cm}}\right)$ using (26). This is the horizontal cross-section of the vertical prism (or planar strip) which forms $\mathcal{E}\left(q_{0}\right)$.

\subsection{Computing the Stability Region}

If $k \geq 6$, the intersection of the $k$ c-obstacle boundaries is generically a zero-dimensional stratum. In this case, $\mathcal{E}\left(q_{0}\right)=\operatorname{int}\left(\mathcal{E S}\left(q_{0}\right)\right)$, and we regard the problem as solved. If $k<6$, then we must know the contact wrench magnitudes $\lambda_{1}, \ldots, \lambda_{k}$ to compute the stability of a pose. If $n_{1}, \ldots, n_{k}$ are linearly independent, which is the generic case for $k \leq 6$, then the horizontal cross-section of $\mathcal{E}\left(q_{0}\right)$ has dimension $a$. Moreover, there is a bijective map between $\mathcal{H}$ and equilibrium locations $\left(x_{\mathrm{cm}}, y_{\mathrm{cm}}\right)$ for the center of mass. Thus we can associate each point in $\mathcal{E}\left(q_{0}\right)$ with a unique point in $\mathcal{H}$ and a unique set of values for $\lambda_{1}, \ldots, \lambda_{k}$, and thereby a uniquely defined relative curvature form. (If $k<6$ but the $n_{1}, \ldots, n_{k}$ are linearly dependent, then the curvature form is not uniquely defined. Here we will treat only the generic case, but a small extension handles the general case.) We assume $k \geq 2$ since we have a complete formal solution for the $k=1$ case.

We can numerically find $(6-k)$ basis twists for the tangent space $T_{q_{0}} S$ that are orthogonal to $n_{1}, \ldots, n_{k}$. It is always possible to choose these basis twists so that no more than two of them have any horizontal torque component. Let $B$ be the matrix whose columns are these 

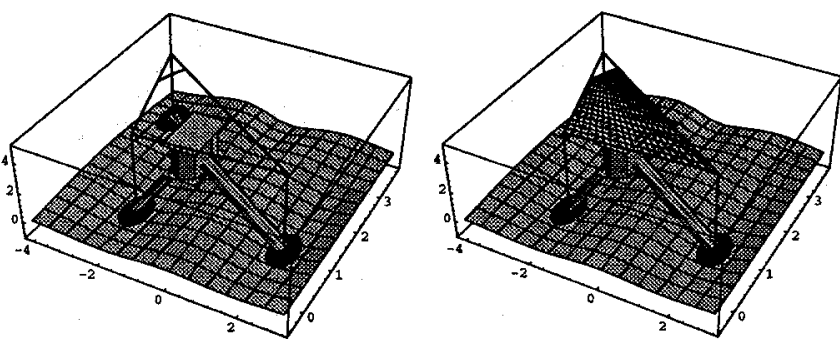

Figure 4: The equilibrium region of this 3-contact pose is a vertical prism. The stability subset is a vertical column whose horizontal cross-section is shaded.

basis vectors. In this basis, $\kappa_{G}=\dot{q}^{T} G \dot{q}$ where $G$ is a $(6-k)$-by- $(6-k)$ matrix $G=B^{T}\left(\sum_{i=1}^{k} \lambda_{i} D n_{i}-D^{2} U\right) B$. The expressions for $D n_{i}$ and $D^{2} U$ are given by equations (2) and (4) respectively. All the terms contained in this matrix are known numerical quantities except for the height of the center of mass $z_{\mathrm{cm}}$, and the quantities $x_{\mathrm{cm}}, y_{\mathrm{cm}}, \lambda_{1}, \ldots, \lambda_{k}$, which are affine functions of $\eta=\left(\eta_{1}, \ldots, \eta_{a}\right)^{T} \in \mathcal{H}$. As a result we can write

$$
G=\mathcal{M}_{0}+\mathcal{M}_{1} \eta_{1}+\cdots+\mathcal{M}_{a} \eta_{a}+B^{T}\left(\begin{array}{cc}
O_{3} & O_{3} \\
O_{3} & \left.\begin{array}{ccc}
z_{\mathrm{cm}} & 0 & 0 \\
0 & z_{\mathrm{cm}} & 0 \\
0 & 0 & 0
\end{array}\right)
\end{array}\right) B
$$

where $\mathcal{M}_{0}, \mathcal{M}_{1}, \mathcal{M}_{2}$ are all constant numerical matrices. To find the stability region we must find values of $\eta \in \mathcal{H}$ and $z_{\mathrm{cm}} \in \mathbb{R}$ such that $G$ is negative-definite. The best way to do this is to consider the sign of the principal minors of $G$. This yields $(6-k)$ inequalities defining $\mathcal{E S}\left(q_{0}\right)$. If we properly order the basis twists and choose them so that at most two have any horizontal torque component, these inequalities will consist of:

1. At most one inequality which is a quadratic function of $z_{\mathrm{cm}}$. The coefficient of $z_{\mathrm{cm}}^{2}$ is a $(5-k)$-degree (at worst, cubic) polynomial in $\eta_{i}$. Other coefficients are $(6-k)$-degree (at worst, quartic).

2. At most one inequality which is a linear function of $z_{\mathrm{cm}}$. The coefficient of $z_{\mathrm{cm}}$ is a $(4-k)$-degree (at worst, quadratic) polynomial in $\eta_{i}$; the remaining term is at worst cubic. These two inequalities containing $z_{\mathrm{cm}}$, if present, define a maximum height for the center of mass at each horizontal point $\left(x_{\mathrm{cm}}, y_{\mathrm{cm}}\right)$ in $\mathcal{E}\left(q_{0}\right)$.

3. Remaining inequalities which do not contain $z_{\mathrm{cm}}$. Whereas the previous inequalities defined a "top" to the stability region, these define the "sides". The worst of these inequalities is at most cubic so there are well-known closed form solutions for the cross-sectional boundaries of $\mathcal{E S}\left(q_{0}\right)$.

\section{Conclusion}

For small numbers of contacts, we have established closed (or nearly closed) form formulas to determine the stability of a frictionless object resting on arbitrarily curved objects. We also showed how to find the region over which the object's center of mass can move while maintaining equilibrium and stable equilibrium. For larger numbers of contacts an efficient algorithm can be used to determine object stability as well as the center of mass locations that ensure equilibrium or stable equilibrium. These results should be useful for automated algorithms that plan the quasi-static locomotion of multi-legged objects over uneven terrain or the manipulation of heavy objects by multiple fingers. In future work we hope to add friction to our analysis.

\section{References}

[1] R. Mason, E. Rimon, and J. Burdick. The stability of heavy objects with multiple contacts. In Proc. IEEE Int. Conf. on Robotics and Automation, May 1995.

[2] M. Erdmann, M. Mason, and J. G. Vanecek. Mechanical parts orienting, the case of a polyhedron on a table. In Proc. IEEE Int. Conf. on Robotics and Automation, Sacramento, CA, May 1991.

[3] J. Wiegley, A. Rao, and K. Goldberg. Computing a statistical distribution of stable poses of a polyhedron. In Proc. Allerton Conf. on Communications, Control and Computing, Oct. 1992.

[4] M. Peshkin and A. Sanderson. Minimization of energy in quasi-static manipulation. IEEE Trans. on Robotics and Automation, 5(1):53-60, Feb. 1989.

[5] D. Kriegman. Computing stable poses of piecewise smooth objects. CVGIP: Image Understanding, 55(2):109-118, Mar. 1992.

[6] J.C. Trinkle, A.O. Farahat, and P.F. Stiller. Secondorder stability cells of a frictionless rigid body grasped by rigid fingers. In Proc. IEEE Int. Conf. on Robotics and Automation, San Diego, CA, May 1994.

[7] E. Rimon and J. W. Burdick. A configuration space analysis of bodies in contact-part ii: $2^{\text {nd }}$ order mobility. Mechanism and Machine Theory, 30(6):913-928, Aug. 1995.

[8] R. Mason, E. Rimon, and J. Burdick. Stable multicontact poses of heavy objects. Technical report, California Institute of Technology, 1996.

[9] J. Ponce, S. Sullivan, J-D. Boissonnat, and J-P. Merlet. On characterizing and computing three- and four-finger force-closure grasps of polyhedral objects. In IEEE Int. Conf. on Robotics and Automation, pages 821-827, Atlanta, GA, May 1993.

[10] Herbert Edelsbrunner. Algorithms in Combinatorial Geometry. Springer-Verlag, 1987. 